\title{
Review of: "Myeloid cell-specific Irf5 deficiency stabilizes atherosclerotic plaques in Apoe mice"
}

\author{
Elias Wieland $^{1}$, Marjo Donners ${ }^{1}$, Pieter Goossens ${ }^{1}$ \\ 1 Maastricht University
}

Potential competing interests: The author(s) declared that no potential competing interests exist.

Interferon regulatory factor (IRF) family members regulate cell development, differentiation and response to pathogens. IRF5 is broadly expressed in immune cells such as B cells, monocytes, macrophages and dendritic cells. A previous study suggested an important role of IRF5 in maintaining CD11 $\mathrm{C}^{+}$macrophages in the atherosclerotic plaque (Seneviratne et al., 2017). However, the model used for that study was ApoeI-Irf5 $\%$ and did not allow to elucidate the cell-specific roles of IRF5 in atherosclerosis. The study by Leipner and colleagues used an atherosclerotic mouse model $\left(\mathrm{Apoe}^{-/-}\right)$combined with a myeloid specific deficiency of Irf5 ( $\mathrm{Lysm}^{\mathrm{Cre} /+} \mathrm{Irf5}{ }^{\mathrm{fl} / \mathrm{fl})}$ to determine macrophage-specific roles of IRF5 in plaque development. Myeloid deficiency of IRF5 led to a stable plaque phenotype, as evidenced by a decreased necrotic core size, lipid and $\left(\mathrm{CD} \mathrm{8}^{+}\right)$macrophage content, while collagen content was increased. Moreover, bone marrow-derived macrophage accumulation, proliferation and lipid uptake was decreased in plaque macrophages (Leipner et al., 2021).

The here used mouse model is effectively targeting macrophages as key players in atherogenesis. Yet, other myeloid cells such as neutrophils and dendritic cells, also shown to be important cell types in atherosclerosis, could be targeted as well by this conditional Irf5 knock-out (Fernández-Ruiz, 2019). The influence of Irf5 deficiency on the whole myeloid population is an important factor to consider when interpreting specific effects of Irf5 deletion in macrophages.

As an elegant starting point for this study, Leipner and colleagues used the murine atherosclerosis single cell data of Winkels et al. and showed that the expression profile of Irf5 in plaque cell populations was predominantly found in macrophages and monocytes while it was also expressed to a minor extent in epithelial and other cell types (Winkels et al., 2018). IRF5 expression in human atherosclerotic plaque macrophages was using the single cell sequencing data from Wirka and colleagues (Wirka et al., 2019). However, no other myeloid populations, e.g. neutrophils or dendritic cells, were included in both figures, hence Irf5 expression in these cells could not be assessed. Moreover, the low number of human cells expressing IRF5 makes it difficult to draw any conclusions from this figure. A comparison with other human plaque single cell RNA sequencing data would be helpful to confirm macrophage-specific expression of IRF5 or reveal its expression profile within other myeloid cells (Fernandez et al., 2019; Wirka et al., 2019; 
Depuydt et al., 2020).

Recent advances on single cell level demonstrated a high heterogeneity and plasticity in human and murine plaque macrophages (Winkels et al., 2018; Fernandez et al., 2019; Lin et al., 2019; Wirka et al., 2019; Depuydt et al., 2020; Williams et al., 2020; Li et al., 2021). Various different phenotypes and subsets have been described so far, and at least 5 subsets of macrophages have been defined in murine aorta, i.e. Inflammatory, Resident-like, Foamy, IFN inducible and Cavity macrophages (Zernecke et al., 2020). Using the single cell data of murine atherosclerotic plaques, expression of Irf5 was observed in inflammatory, resident-like and foamy subsets (Winkels et al., 2018). While these findings were confirmed in similar subsets of human macrophages, IRF5 expression in the large heterogeneity of other macrophage subsets has not been investigated yet.

Interestingly, deficiency of Irf5 seemed to impair in vitro macrophage polarization towards the proinflammatory (M1) phenotype, while it also had an impact on non-inflammatory genes such as Abcal (lipid efflux), Cd36 (lipid uptake), Mertk (efferocytosis) and Arg1 (pro-fibrotic). Similarly, the gene expression profiles of aortas from Irf5 deficient mice showed a relative increase in Mertk, Tgfb, Cd206 and Arg1 compared to aortas from mice with normal Irf5 expression. These data show that Irf5 deficiency in myeloid cells in vivo stabilizes the plaque with a concomitant anti-inflammatory and pro-fibrotic (i.e. M2like) gene expression pattern. In line with this, Irf5 expression was previously associated with macrophage polarization towards a pro-inflammatory phenotype and the suppression of an anti-inflammatory polarization (Krausgruber et al., 2011). Notably, Irf5 was found to be expressed in $\mathrm{NOS}^{+} \mathrm{CD}^{2} 8^{+}$and in $\mathrm{CD}_{11 \mathrm{C}^{+}}$subsets, suggesting a heterogeneous distribution of Irf5 expression among macrophage subpopulations (Krausgruber et al., 2011; Seneviratne et al., 2017).

To elucidate the mechanism how Irf5 deficiency decreased $\mathrm{CD} 68^{+}$cell content in the plaque, a chimeric mouse model was created reconstituting irradiated $A p o e^{-/-}$mice with a mixture of Apoe ${ }^{-/-}$CD45.1 $1^{+}$WT and $\mathrm{Apoe}^{-/-} \mathrm{CD} 45.2^{+}$Irf5 ${ }^{-/-}$bone marrow to replenish plaque macrophages. It was observed that, while monocyte recruitment was not affected by Irf5, the local proliferation of Irf5 deficient macrophages in plaque was decreased. Moreover, ex vivo differentiation towards macrophages was decreased in Irf5 deficient circulating and splenic monocytes. Thus, Irf5 plays a role in local macrophage differentiation and proliferation rather than in monocyte influx.

In summary, the article by Leipner and colleagues provides evidence for an important role of Irf5 in plaque macrophage accumulation and phenotypic differentiation/polarization.

Recent advances and state of the art methods such as CyTOF, or more affordable techniques such as multiplex immunofluorescence, can help to reveal which cell types, subsets or phenotypes express Irf5 in 
their specific spatial context/microenvironment, particularly in the complexity of human atherosclerosis. Furthermore, functional analyses could contribute to solidify the role of Irf5 deletion in macrophage polarization and atherogenic functions.

References

Depuydt, M. A. C. et al. (2020) 'Microanatomy of the Human Atherosclerotic Plaque by Single-Cell Transcriptomics', Circulation research, 127(11), pp. 1437-1455. doi: 10.1161/CIRCRESAHA.120.316770. Fernández-Ruiz, I. (2019) 'Neutrophil-driven SMC death destabilizes atherosclerotic plaques', Nature Reviews Cardiology 2019 16:8, 16(8), pp. 455-455. doi: 10.1038/s41569-019-0214-1.

Fernandez, D. M. et al. (2019) 'Single-cell immune landscape of human atherosclerotic plaques', Nature Medicine, 25(10), pp. 1576-1588. doi: 10.1038/s41591-019-0590-4.

Krausgruber, T. et al. (2011) 'IRF5 promotes inflammatory macrophage polarization and T H 1-T H 17 responses', nature immunology, 12. doi: 10.1038/ni.1990.

Leipner, J. et al. (2021) 'Myeloid cell-specific Irf5 deficiency stabilizes atherosclerotic plaques in Apoe-/mice', Molecular Metabolism, 53. doi: 10.1016/J.MOLMET.2021.101250.

Li, F. et al. (2021) 'Single-cell RNA-seq reveals cellular heterogeneity of mouse carotid artery under disturbed flow', Cell Death Discovery 2021 7:1, 7(1), pp. 1-14. doi: 10.1038/s41420-021-00567-0. Lin, J. Da et al. (2019) 'Single-cell analysis of fate-mapped macrophages reveals heterogeneity, including stem-like properties, during atherosclerosis progression and regression', JCI Insight, 4(4). doi: 10.1172/JCI.INSIGHT.124574.

Seneviratne, A. N. et al. (2017) 'Interferon Regulatory Factor 5 Controls Necrotic Core Formation in Atherosclerotic Lesions by Impairing Efferocytosis', Circulation, 136(12), pp. 1140-1154. doi:

10.1161/CIRCULATIONAHA.117.027844.

Williams, J. W. et al. (2020) 'Limited proliferation capacity of aorta intima resident macrophages requires monocyte recruitment for atherosclerotic plaque progression', Nature immunology, 21(10), p. 1194. doi: 10.1038/S41590-020-0768-4.

Winkels, H. et al. (2018) 'Atlas of the immune cell repertoire in mouse atherosclerosis defined by single-cell RNA-sequencing and mass cytometry', Circulation Research, 122(12), pp. 1675-1688. doi: 10.1161/CIRCRESAHA.117.312513.

Wirka, R. C. et al. (2019) 'Atheroprotective roles of smooth muscle cell phenotypic modulation and the TCF21 disease gene as revealed by single-cell analysis', Nature Medicine 2019 25:8, 25(8), pp. 1280-1289. doi: 10.1038/s41591-019-0512-5.

Zernecke, A. et al. (2020) 'Meta-Analysis of Leukocyte Diversity in Atherosclerotic Mouse Aortas', Circulation research, 127(3), pp. 402-426. doi: 10.1161/CIRCRESAHA.120.316903. 